\title{
Artificial neural networks for solving elliptic differential equations with boundary layer
}

\author{
Dongfang Yuan ${ }^{1}$, Wenhui Liu ${ }^{1}$, Yongbin $\mathrm{Ge}^{2}$, Guimei Cui ${ }^{1}$, $\mathrm{Lin}^{\mathrm{Shi}^{1}}$, and Fujun $\mathrm{Cao}^{1}$ \\ ${ }^{1}$ Inner Mongolia University of Science and Technology \\ ${ }^{2}$ Ningxia University
}

December 26, 2020

\begin{abstract}
In this paper, we consider the artificial neural networks for solving the differential equation with boundary layer, in which the gradient of the solution changes sharply near the boundary layer. The solution of the boundary layer problems poses a huge challenge to both traditional numerical methods and artificial neural network methods. By theoretical analyzing the changing rate of the weights of first hidden layer near the boundary layer, a mapping strategy is added in traditional neural network to improve the convergence of the loss function. Numerical examples are carried out for the $1 \mathrm{D}$ and $2 \mathrm{D}$ convection-diffusion equation with boundary layer. The results demonstrate that the modified neural networks significantly improve the ability in approximating the solutions with sharp gradient.
\end{abstract}

\section{Hosted file}

NN4PDE.pdf available at https://authorea.com/users/385338/articles/500814-artificial-neuralnetworks-for-solving-elliptic-differential-equations-with-boundary-layer

\section{Hosted file}

NN4PDE.rar available at https://authorea.com/users/385338/articles/500814-artificial-neuralnetworks-for-solving-elliptic-differential-equations-with-boundary-layer 